\title{
IR Spectroscopy of High-Redshift Quasars
}

Jill Bechtold, Joseph Shields, Marcia Rieke, Peiwen Ji, ${ }^{1}$ and Jennifer Scott

Steward Observatory, University of Arizona, Tucson, AZ 85721, USA

Olga Kuhn ${ }^{2}$ and Martin Elvis

Harvard-Smithsonian Center for Astrophysics, Cambridge, MA 02138, $U S A$

Richard Elston

Cerro Tololo Inter-American Observatory, La Serena, Chile

\begin{abstract}
We have obtained near-IR, optical, and soft X-ray spectrophotometry for a number of bright quasars with $2 \lesssim z \lesssim 4$. The observed ratios of $\mathrm{Ly} \alpha / \mathrm{H} \beta$ in this sample are smaller than Case $\mathrm{B}$, and span a similar range to those seen at lower redshift. A correlation between the line width of $\mathrm{H} \beta$ and $\mathrm{X}$-ray spectral index found for low-redshift PG quasars is also seen for the high-redshift objects.
\end{abstract}

\section{Introduction}

Recent advances in near-IR spectroscopic capabilities have made possible observations of the rest-frame optical and UV radiation from high-redshift quasars with unprecedented spectral resolution and signal-to-noise ratio. We have observed a number of $z \approx 2$ and $z \approx 3$ quasars in the near-IR with FSPEC and GESPEC at the MMT, OSIRIS at the CTIO 4m, and CRSP at the KPNO $4 \mathrm{~m}$. Optical spectrophotometry was obtained nearly simultaneously with the $2.3-\mathrm{m}$ Bok Telescope, FLWO 1.5-m and CTIO 1.5-m telescopes. Details are presented by Bechtold et al. 1994a, Kuhn et al. 1995, and Kuhn (1996), and in other work in preparation. One of the goals of this study is to examine empirical correlations between line and continuum properties which have been observed for objects at low redshift and see if they also hold for more luminous, high-redshift objects. Here we present two such correlations.

\footnotetext{
${ }^{1}$ Also Department of Mathematics and Physical Science, National Natural Science Foundation of China, Beijing, China.

${ }^{2}$ Current Address: Observatorio Astronomico de Nacional, Ensenada, Mexico.
} 


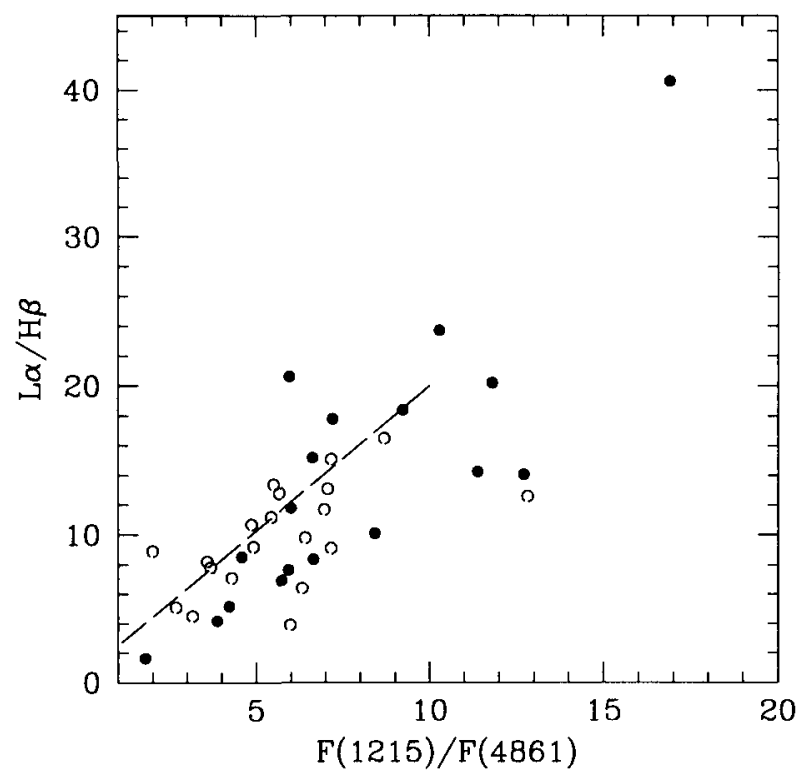

Figure 1. $\quad \mathrm{Ly} \alpha / \mathrm{H} \beta$ versus ratio of continuum flux at adjacent points. Open circles are intermediate-redshift, radio-loud quasars from Netzer et al. (1995) and closed circles are high redshift quasars. Dashed line indicates reddening vector, for a Galactic reddening law, and dust located external to both the line-emitting and continuum-emitting regions.

\section{2. $\operatorname{Ly} \alpha / \mathbf{H} \beta$}

Baldwin (1977) first showed that the ratio of $\mathrm{Ly} \alpha / \mathrm{H} \beta$ in the broad emission lines of quasars is lower than predictions of Case $\mathrm{B}$ recombination theory by as much as a factor of 10 . This has been interpreted either as a result of reddening, or trapping of Ly $\alpha$ photons and collisional excitation in regions of high optical depth, which enhances the Balmer lines relative to Ly $\alpha$ (e.g., Davidson \& Netzer 1979). Netzer et al. (1995) and Wills et al. (1995) present measurements of the ratio of $\mathrm{Ly} \alpha / \mathrm{H} \beta$ for their sample of intermediate-redshift, radio-loud quasars observed with FOS on HST. In Fig. 1, we show this ratio versus $F(1215) / F(4861)$, the ratio of the continuum fluxes adjacent to the emission lines (cf. Netzer et al.'s Fig. 6).

The high-redshift quasars of our sample appear to have $\mathrm{Ly} \alpha / \mathrm{H} \beta$ ratios similar to those of the HST sample, and lie along the same correlation with continuum flux ratio. Figure 1 can be explained if all objects have near-Case $B \operatorname{Ly} \alpha / \mathrm{H} \beta$ ratios of $\sim 20$ and a modest amount of foreground dust $(E(B-V) \lesssim 0.3)$, which 


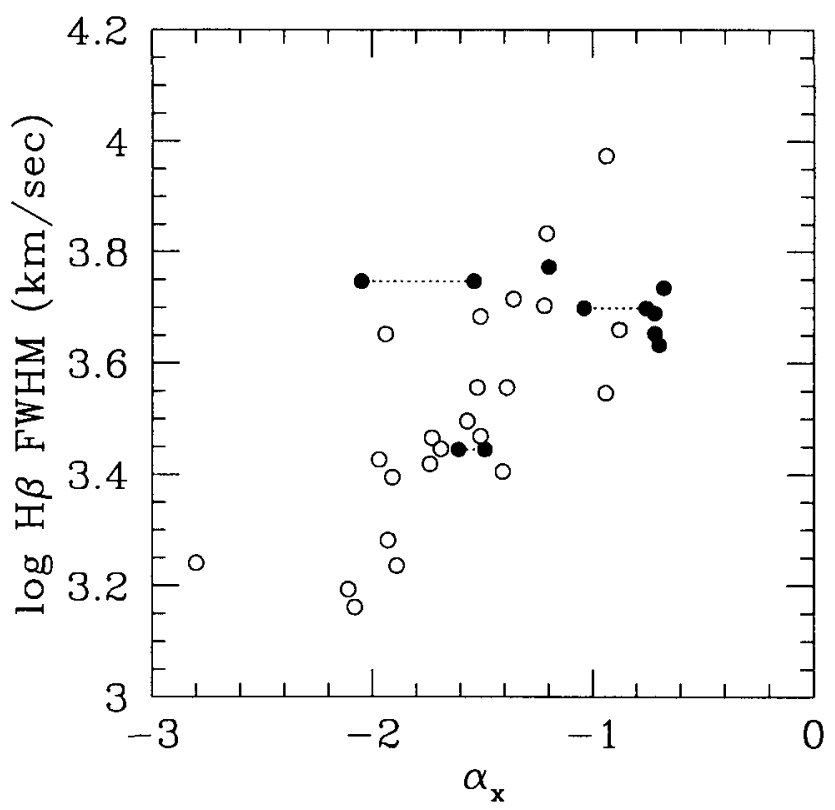

Figure 2. Line width of $\mathrm{H} \beta$ versus $\mathrm{X}$-ray spectral index. Open circles are PG quasars from Laor et al. $(1994,1996)$ and filled circles are high-redshift quasars. Dotted lines connect the range of allowed X-ray spectral indices for objects for which only PSPC colors are available (see Bechtold et al. 1994b).

reddens both the continuum and emission lines by the same amount. Qualitatively one expects $\operatorname{Ly} \alpha / \mathrm{H} \beta$ to be lower in luminous quasars which have larger UV/X-ray continuum flux ratios, compared to fainter objects which have harder continua. Here, the radio-loudness of low-redshift sample may counteract this effect, since the high-redshift quasars are a mix of radio-loud and radio-quiet objects.

\section{3. $\mathrm{H} \beta$ Line Width versus $\alpha_{x}$}

Laor et al. $(1994,1996)$ found a correlation between $\mathrm{H} \beta$ line width and soft $\mathrm{X}$ ray spectral index for the low-redshift PG quasars observed with ROSAT. While the origin of this correlation is not clear (see the discussion in Laor et al. 1996 and references therein), it is of interest to see whether it holds as well for the much more luminous high-redshift objects. Figure 2 shows the result for the PG quasars from Laor et al. (1996) and the high-redshift quasars which have X-ray spectral indices or colors measured by $A S C A$ or the ROSAT PSPC (Bechtold 
et al. 1994a,b, Elvis et al. 1994, Serlemitsos et al. 1994, Bechtold et al. 1996). Note that the spectral indices (here defined as $f_{E} \propto E^{\alpha_{x}}$ ) for the high-redshift quasars correspond to harder rest-frame $\mathrm{X}$-ray energies than for the low-redshift PG quasars observed with ROSAT. The high-redshift objects lie along the same relation, indicating that the origin must be relatively independent of luminosity.

Acknowledgments. JB acknowledges the support of NASA grants NAG52080 and NAG5-2962, and NSF grant AST-9058510. Observations reported here were obtained at the Multiple Mirror Telescope Observatory, a facility operated jointly by the University of Arizona and the Smithsonian Institution.

\section{References}

Baldwin, J. A. 1977, MNRAS, 178, 67P.

Bechtold, J., Elvis, M., Fiore, F., Kuhn, O., Cutri, R. M., McDowell, J. C., Rieke, M., Siemiginowska, A., Wilkes, B. J. 1994a, AJ, 108, 374.

Bechtold, J., Elvis, M., Fiore, F., Kuhn, O., Cutri, R. M., McDowell, J.C., Rieke, M., Siemiginowska, A., Wilkes, B.J. 1994b, AJ, 108, 759.

Bechtold, J., et al. 1996, in preparation.

Davidson, K., and Netzer, H. 1979, Rev. Mod. Phys., 51, 715.

Elvis, M., Matsuoka, M., Siemiginowska, A., Fiore, F., Mihara, T., Brinkmann, W. 1994, ApJ, 436, L55.

Kuhn, O. 1996, Ph.D. thesis, Harvard University.

Kuhn, O., Bechtold, J., Cutri, R., Elvis, M., Rieke, M. 1995, ApJ, 438, 643.

Laor, A., Fiore, F., Elvis, M., Wilkes, B.J., McDowell, J.C. 1994, ApJ, 435, 611.

Laor, A., Fiore, F., Elvis, M., Wilkes, B. J., McDowell, J. C., 1996, preprint.

Netzer, H., Brotherton, M.S., Wills, B.J., Han, M., Wills, D., Baldwin, J. A., Ferland, G. J., Browne, I. W. A. 1995, ApJ, 448, 27.

Serlemitsos, P., Yaqoob, T., Ricker, G., Woo, J., Kunieda, H., Terashima, Y., Iwasawa, K. 1994, PASJ, 46, L43.

Wills, B. J., Thompson, K.L., Han, M., Netzer, H., Wills, D., Baldwin, J. A., Ferland, G. J., Browne, I. W. A., Brotherton, M. S. 1995, ApJ, 447, 139. 